\title{
Inanspruchnahme
}

\section{eines Beratungsangebots zu exzessivem Medienkonsum}

\author{
Nora M. Laskowski ${ }^{1,2}$, Alexandra Ernst ${ }^{1}$, Viktoria Meyer ${ }^{1}$, Tobias Trillmich³ und Astrid Müller \\ Klinik für Psychosomatik und Psychotherapie, Medizinische Hochschule Hannover \\ Abteilung für Klinische Psychologie, Psychotherapie und Diagnostik, Institut für Psychologie, Technische Universität Braunschweig \\ Niedersächsische Landesstelle für Suchtfragen, Hannover
}

\begin{abstract}
Zusammenfassung: Zielsetzung: Von 2017 bis 2020 wurde in Niedersachsen das Projekt „re:set! - Beratung bei exzessivem Medienkonsum“ durchgeführt. Der Beitrag adressiert die Inanspruchnahme des Beratungsangebots, die Soziodemographie und die präferierten Anwendungen der Betroffenen sowie die Prävalenz von Internetnutzungsstörungen (INS) in dieser Gruppe. Methodik: Inanspruchnahme und Soziodemographie der Betroffenen wurden anhand eines Dokumentationsbogens erhoben. Die Kurzform der „Assessment for Internet and Computer Game Addiction Scale“ diente der Erhebung der Symptome einer INS. Ergebnisse: 90,2\% der 1053 beratungsaufsuchenden Personen waren männlich, das mittlere Alter lag bei 21.65 Jahren. Mit 49,3\% wurden Online-Spiele am häufigsten genutzt. Online-Spiele/-Erotikangebote wurden eher von männlichen, Online-Kaufportale/-Communities eher von weiblichen Betroffenen genutzt. Bei 64,6\% der Betroffenen zeigten sich Hinweise auf eine INS. Schlussfolgerungen: Suchtberatungsstellen können erste Anlaufstellen für Menschen mit exzessivem Medienkonsum oder INS und deren Angehörige sein.
\end{abstract}

Schlüsselwörter: Suchtberatung, Suchthilfe, internetbezogene Störungen, Internetnutzungsstörungen, exzessiver Medienkonsum

Utilization of a Counselling Service for Excessive Media Use

Abstract: Purpose: The project "re:set! - Counselling for excessive media use" was carried out in Lower Saxony (Germany [Niedersachsen]) between 2017 and 2020. The present article is concerned with the utilization of this specific counselling service, the sociodemographic characteristics, the preferred internet applications of those using the service, and the prevalence of internet use disorders (IUD) in this sample. Methods: The utilization of this counselling service and sociodemographic variables of the clients were examined using a questionnaire developed for this project. The short version of the "Assessment for Internet and Computer Game Addiction Scale" was administered to all clients to assess the symptoms of IUD. Results: Counselling was provided to 1053 mainly male (90.2\%) individuals, whose average age was 21.65 years. Onlinegames were used most frequently (49.3\%). Online-games/-pornography were more likely to be used by males, whereas females tended to prefer shopping websites and social networks. A large proportion (64.6\%) of service users in the present sample were at risk of IUD. Conclusion: Centers for addiction counselling and prevention can serve as first contact point for individuals with excessive media use or IUD and their relatives.

Keywords: addiction counselling, addiction aid, internet-related disorders, internet-use disorder (IUD), excessive media use

\section{Einführung}

Die Störung durch Computerspielen (Kategorie 6C51: „Gaming Disorder") wurde mit der 11. Version der Internationalen Klassifikation psychischer Störungen (ICD-11; WHO, 2019) erstmals in das Klassifikationssystem aufgenommen. Zugeordnet wurde sie der neuen Kategorie der „Störungen durch abhängige Verhaltensweisen“ („Disorders due to addictive behaviors"; WHO, 2019), in der auch die Störung durch Glücksspielen gelistet ist. Für beide Störungen sind eine vorwiegend offline (,predominantly offline“) und eine vorwiegend online (,predominantly online“) ausgeführte Variante vorgesehen. Somit haben erstmals zwei spezifische Internetnutzungsstörungen (INS) Eingang in die ICD gefunden, während andere nicht berücksichtigt wurden. In der Fachliteratur werden vor allem die pathologische Nutzung von Online-Kaufportalen, Online-Pornografie oder sozialen Netzwerken als weitere spezifische INS diskutiert (Brand et al., 2020). Angesichts der noch unzureichenden Versorgungsstruktur werden in Fachkreisen Interventionen bezogen auf Prävention, Beratung und Behandlung aller INS gefordert, also auch derjenigen, die 
noch nicht in den Klassifikationssystemen abbildbar sind (Brand et al., 2020; Müller et al., 2019; Rumpf et al., 2016).

Bereits 2018 wurden Beratungsaufsuchende mit exzessivem Medienkonsum in die Suchthilfestatistik aufgenommen und stellten etwa 1\% aller Beratungsaufsuchenden im Suchthilfesystem dar (Dauber, Specht, Künzel, PfeifferGerschel \& Braun, 2019). Während manche Fachstellen für Suchtberatung und -prävention über eine sehr gute Expertise im Bereich INS verfügen, gilt dies längst nicht für alle. Petersen, Hanke, Bieber, Mühleck und Batra (2017) identifizierten deutschlandweit 253 Angebote für Beratung oder Behandlung von INS, 26 Einrichtungen davon in Niedersachsen. Flächendeckende Beratungsangebote für Menschen mit INS fehlen, sodass dringender Nachholbedarf bezogen auf die Versorgungsstrukturen besteht (Wedegärtner \& Wedegärtner, 2010). Dabei ist bekannt, dass die Kontaktaufnahme für Menschen mit INS sowie deren Angehörige über das Versorgungsnetzwerk der Fachstellen für Suchtberatung und -prävention erfolgt (Petersen et al., 2017; Wedegärtner \& Wedegärtner, 2010). Aus diesem Grund wurde das in Niedersachsen angesiedelte Projekt „re:set! - Beratung bei exzessivem Medienkonsum" initiiert. Ziel des re:set!-Projektes war die Einrichtung eines qualifizierten Beratungsangebotes für Betroffene mit einem exzessiven Medienkonsum oder ersten Symptomen einer INS und deren Angehörige. Auch die Vernetzung der Einrichtungen sollte gefördert werden, wie von Expert_innen lange gefordert (Petersen \& Thomasius, 2010). Das Projekt wurde vom Niedersächsischen Ministerium für Soziales, Gesundheit und Gleichstellung gefördert. Die Koordination des Projektes lag in den Händen der Niedersächsischen Landesstelle für Suchtfragen (NLS) und die wissenschaftliche Begleitung erfolgte durch die Arbeitsgruppe „Substanzungebundene Abhängigkeitserkrankungen" der Klinik für Psychosomatik und Psychotherapie der Medizinischen Hochschule Hannover (MHH). Von 2017 bis 2020 nahmen insgesamt 16 Fachstellen für Sucht und Suchtprävention an dem Projekt teil (im ersten Projektjahr zunächst 8, danach insgesamt 16 Fachstellen). Diese Fachstellen verteilten sich auf folgende niedersächsische Standorte: Achim/Verden, Braunschweig, Cuxhaven, Delmenhorst, Goslar, Göttingen, Hannover, Hildesheim, Leer, Lüneburg, Meppen, Oldenburg, Osnabrück, Salzgitter, Twistringen, Wolfsburg.

Die beteiligten Fachkräfte wurden durch regelmäßige Fortbildungen beim Aufbau des Beratungsangebotes unterstützt, das ausführlich dokumentiert wurde (Müller, Ernst, Meyer, Jones \& Laskowski, 2020). Auch ein regelmäßiger Austausch inkl. spezifischer Fallbesprechungen in regionalen Netzwerktreffen (Nord-West- bzw. Süd-OstNiedersachsen) fand statt. So sollte das Projekt die Bildung eines nachhaltigen medienspezifischen Netzwerkes und einer spezifischen Beratungsstruktur fördern. Zudem wurden gemeinsam mit den Fachkräften „Empfehlungen zur Implementierung eines Beratungsangebots bei exzessivem Medienkonsum und internetbezogenen Störungen" entwickelt und publiziert (Müller, Meyer, Schwenk \& Laskowski, 2019; Laskowski, Meyer, Trillmich, Ernst \& Müller, 2020). Darüber hinaus wurde eine umfangreiche, praxisnahe Informations- und Materialsammlung für die Beratung bei exzessivem Medienkonsum erarbeitet (Müller, Meyer, Ernst \& Laskowski, 2020), die inzwischen auf Anfrage bei der NLS erhältlich ist (Kontakt: trillmich@nlsonline.de).

Ziel der wissenschaftlichen Begleitung des Projekts war es, die Inanspruchnahme des neuen Beratungsangebots zu explorieren. Darüber hinaus sollten die soziodemographischen Charakteristika und präferierten Internetanwendungen der Beratungsaufsuchenden erhoben und Erkenntnisse zur Prävalenz von INS in dieser Gruppe gewonnen werden. Zu erwähnen ist, dass laut Vorgabe des Förderers pro Jahr mindestens 50 Beratungstermine pro Fachstelle erwartet wurden.

Verschiedene Studien zeigten, dass männliche und weibliche Betroffene sich hinsichtlich der genutzten Internetapplikationen unterscheiden (Petersen \& Thomasius, 2010; Petersen et al., 2017). Eine problematische Nutzung von Computerspielen (Rehbein, Kliem, Baier, Mößle \& Petry, 2015; Dufour et al., 2016), Online-Erotikangeboten (Dufour et al., 2016; Ballester-Arnal, Castro Calvo, GilLlario \& Gil-Julia, 2017) sowie Glücksspielen (Petry, Stinson \& Grant, 2005; McCormack, Shorter \& Griffiths, 2014) betrifft eher männliche Personen. Suchtartiges Kaufen (Maraz, Griffiths \& Demetrovics, 2016) und die problematische Nutzung von sozialen Netzwerken (Dufour et al., 2016; Ostendorf, Wegmann \& Brand, 2020) scheinen eher weibliche Phänomene zu sein. Basierend auf der Literatur wurde die Hypothese aufgestellt, dass sich eben diese Geschlechtsunterschiede auch in der re:set!-Stichprobe finden lassen.

\section{Methodik}

\section{Erhebungsinstrumente}

Zur Dokumentation der Beratungen wurde zu Beginn des Projektes ein Dokumentationsbogen erstellt, der die Inanspruchnahme des Angebots standardisiert messen sollte und zudem die Möglichkeit bot, die Soziodemographie der Betroffenen und deren bevorzugtes Online-Angebot abzubilden. Die Erhebung erfolgte pseudonymisiert anhand einer Identifikationsnummer (ID), die es möglich machte, Folgetermine derselben Person zuordnen zu können. Der Dokumentationsbogen wurde für jedes Beratungsgespräch 
von der beratenden Fachkraft ausgefüllt. Dabei war es irrelevant, ob der/die Betroffene selbst oder eine andere stellvertretende Person (z.B. Angehörige, Multiplikator_in) beraten wurde. Es wurde notiert, wer genau bei dem Termin zugegen war. Die Angaben im Dokumentationsbogen bezogen sich immer auf die betroffene Person.

Um die klinische Relevanz des exzessiven Medienkonsums abzuschätzen, wurden die Betroffenen zudem gebeten, die Kurzform der „Assessment for Internet and Computer Game Addiction Scale" (AICA-S short; Fachverband Medienabhängigkeit e.V., 2010) zur Messung von Symptomen einer INS auszufüllen. Dieser Fragebogen wurde ausschließlich von den Betroffenen selber ausgefüllt (d.h., nicht von Angehörigen o. Ä.). Die AICA-S short (Fachverband Medienabhängigkeit e.V., 2010) ist ein Selbsteinschätzungsinstrument, das zunächst die Nutzungshäufigkeit folgender Internetapplikationen auf einer Skala von 0 (= nie) bis 3 (= sehr oft) erhebt: Online-Spiele, Einkaufen, Chatten/Austausch in Foren, E-Mails, Online-Erotikangebote, Online-Glücksspiele, Online-Communities, Informations-recherche, Video- oder Streamingportale, andere Internetspiele, allgemeines Surfen, anderes. Anhand von 6 weiteren Items wird die Gefährdung für eine INS bezogen auf das bevorzugte Online-Angebot erhoben. Die Items werden auf einer Skala von 0 (= gar nicht/nie) bis 4 (= sehr stark/sehr oft) beantwortet. AICA-S short Summenwerte $\geq 11$ weisen auf eine moderate und Summenwerte $\geq 15$ auf eine schwere INS hin (Fachverband Medienabhängigkeit e.V., 2010). Zusätzlich enthält die AICA-S short noch eine offene Frage nach der durchschnittlichen Nutzungszeit des bevorzugten Online-Angebots pro Wochentag. Cronbachs Alpha der AICA-S short wird in Validierungsstudien zwischen 0.83-0.85 angegeben (Wölfling, Beutel \& Müller, 2016), in der vorliegenden Stichprobe liegt es bei 0.88. Die AICA-S short ist auf der Website des Fachverbandes Medienabhängigkeit e.V. verfügbar: https://www.fv-medienabhaengigkeit.de/publikationen/publikationen-des-ver bands/.

\section{Statistische Analysen}

Statistische Analysen erfolgten mit SPSS Version 26 (IBM Corp., Armonk, NY, USA). Deskriptive Angaben enthalten absolute und relative Häufigkeiten sowie Mittelwerte $(M W)$ und Standardabweichungen $(S D)$. Gruppenvergleiche für kategoriale Variablen wurden mittels $\chi^{2}$-Tests bzw. Fisher-Exakt-Tests (bei mehr als 20\% Zellhäufigkeiten mit Werten $<5$ ) überprüft. Für dichotome Variablen wurde Phi $(\varphi)$, für Variablen mit mehr als zwei Kategorien Cramers $\mathrm{V}(V)$ als Effektstärke bestimmt. $V / \varphi$-Werte $\leq 0.1$ zeigen einen kleinen, 0.3 einen mittleren und $\geq 0.5$ einen großen Effekt an (Cohen, 1988). Das Alter als metrische
Variable war nicht normalverteilt (Kolmogorov-SmirnovTest: $p<0.0001$ ), sodass hier der Mann-Whitney-U-Test und das partielle Eta-Quadrat $\left(\eta_{\mathrm{p}}{ }^{2}\right)$ als Effektstärke für die Gruppenvergleiche herangezogen wurden. Dabei werden $\eta_{\mathrm{p}}{ }^{2}$-Werte $<0.06$ als kleiner, 0.06-0.14 als mittlerer und $>0.14$ als großer Effekt angesehen (Cohen, 1988). Angesichts der multiplen Vergleiche wurde das Signifikanzniveau auf $1 \%$ festgelegt, sodass $p$-Werte $<0.01$ als statistisch signifikant gewertet wurden.

\section{Ergebnisse}

\section{Beschreibung der Stichprobe}

Die Ergebnisse beziehen sich auf den Dokumentationszeitraum 15.03.2017 bis 31.01.2020. Im Rahmen dieses Zeitfensters wurden insgesamt 1053 Betroffene in 3000 Terminen beraten. Hierbei ist zu beachten, dass im ersten Projektjahr zunächst 8, ab dem 2. Projektjahr dann 16 Fachstellen Teil des Projektes waren. Die Stichprobe umfasste 948 (90,2\%; 2 fehlende Angaben) männliche Betroffene. Insgesamt waren $55,7 \%$ der Betroffenen über 18 Jahre alt $(n=583 ; 7$ fehlende Angaben). Das durchschnittliche Alter lag bei 21.65 Jahre $(S D=10.25 ; M d n=19$; Range 7-76). Weitere soziodemographische Variablen finden sich in Tabelle 1.

\section{Beschreibung der Beratungstermine}

Durchschnittlich hat jede der 16 re:set!-Fachstellen 66 Betroffene in die medienspezifische Beratung (Range 25-101) eingeschlossen und dabei im Mittel 188 medienspezifische Beratungstermine (Range 61-338) absolviert. Pro betroffener Person wurden im Schnitt 2.9 Beratungstermine wahrgenommen $(S D=2.1)$, wobei $48,9 \%$ der Beratungsaufsuchenden $(n=515)$ nur für ein Erstgespräch kamen. Die Anwesenheit bei den Erstterminen gliedert sich zu je einem Drittel auf in Betroffene mit Begleitung $(n=310 ; 29,4 \%)$, Betroffene ohne Begleitung $(n=358$; $34,0 \%$ ) und Personen ohne Betroffene (z. B. Angehörige; $n=385 ; 36,6 \%)$. Bei Betroffenen in Begleitung waren die Mütter am häufigsten mit anwesend $(n=137 ; 44,2 \%)$ und auch bei Gesprächen ohne die Anwesenheit der Betroffenen waren die Mütter am häufigsten in der Fachstelle vorstellig $(n=213 ; 55,3 \%)$.

Die Termine $(N=3000)$ fanden hauptsächlich persönlich $(n=2742 ; 91,4 \%)$, gefolgt von telefonisch $(n=198$; $6,6 \%)$ und via E-Mail $(n=45 ; 1,5 \%)$ statt. Nur 15 Gespräche $(0,5 \%)$ fanden auf anderen Wegen statt (z.B. per Brief). 
Tabelle 1. Beschreibung der Stichprobe

\begin{tabular}{|c|c|}
\hline Variable & $n(\%)$ \\
\hline Muttersprache; $N$ & 1043 \\
\hline Deutsch & $986(94,5)$ \\
\hline Russisch & $12(1,2)$ \\
\hline Polnisch & $7(0,7)$ \\
\hline Arabisch & $6(0,6)$ \\
\hline Türkisch & $6(0,6)$ \\
\hline Andere ${ }^{a}$ & $26(2,5)$ \\
\hline Partnerschaft; $N$ & 1031 \\
\hline Mit Partner_in & $194(18,8)$ \\
\hline Ohne Partner_in & $837(81,2)$ \\
\hline Wohnsituation; $N$ & 1435 (inkl. Mehrfachnennungen) \\
\hline Allein lebend & $138(9,6)$ \\
\hline Mit beiden Eltern lebend & $376(26,2)$ \\
\hline Mit einem Elternteil lebend & $223(15,5)$ \\
\hline Mit Geschwistern lebend & $333(23,2)$ \\
\hline Mit Partner_in lebend & $120(8,4)$ \\
\hline Andere $^{b}$ & $245(17,1)$ \\
\hline Ausbildungssituation; $N$ & 1040 \\
\hline Aktuell in Ausbildung/Schule/Universität & $653(62,8)$ \\
\hline Davon regelmäßiger Besuch der Ausbildungsstätte ${ }^{c}$ & $496(76,0)$ \\
\hline Davon unregelmäßiger Besuch der Ausbildungsstätte ${ }^{c}$ & $121(18,5)$ \\
\hline Nicht in Ausbildung/Schule/Universität & $387(37,2)$ \\
\hline Schulabschluss; $N$ & 519 \\
\hline Abitur & $178(34,3)$ \\
\hline Realschulabschluss & $198(38,2)$ \\
\hline Hauptschulabschluss & $137(26,4)$ \\
\hline Sonderschulabschluss & $6(1,2)$ \\
\hline Erwerbstätigkeit; $N$ & 348 \\
\hline Arbeitssuchend & $140(36,5)$ \\
\hline Teilzeit & $22(5,7)$ \\
\hline Vollzeit & $135(35,2)$ \\
\hline Andere $^{d}$ & $87(22,7)$ \\
\hline
\end{tabular}

Anmerkungen: andere Muttersprachen: Französisch ( $n=4 ; 0,4 \%)$, Albanisch $(n=3 ; 0,3 \%$ ), je $n=2 ; 0,2 \%$ : Spanisch, Serbokroatisch, Rumänisch, Afghanisch, je $n=1 ; 0,1 \%$ : Vietnamesisch, Ukrainisch, Niederländisch, Moldawisch, Mexikanisch, Mazedonisch, Litauisch, Kurdisch, Italienisch, Filipino, Chinesisch; ${ }^{b}$ Andere Wohnsituation: Mit einem Elternteil und Partner_in $(n=67 ; 4,7 \%)$, Mutter und Vater im Wechsel $(n=16 ; 1,1 \%)$, mit eigenen Kindern $(n=50 ; 3,5 \%)$,

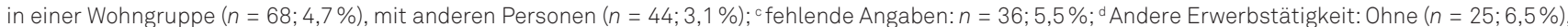
Elternzeit $(n=3 ; 0,8 \%)$, Ruhestand $(n=15 ; 3,9 \%)$, Arbeitsunfähigkeit $(n=19 ; 4,9 \%)$, Sonstige ohne Angabe $(n=25 ; 6,5 \%)$.

\section{Zugangsweg und Weitervermittlung}

Als Zugangsweg ( $N=1144$ inkl. Mehrfachnennung) wurde am häufigsten eine Internetrecherche angegeben $(n=426$; $37,2 \%$ ), gefolgt von einer Vermittlung durch andere (nicht medienbezogene) Beratungsangebote/-stellen $(n=225$; 19,7\%). Insgesamt gaben 87 Betroffene $(8,3 \%)$ an, dass sie zuvor bereits an anderer Stelle bezüglich ihres exzessiven Medienkonsums beraten worden waren. Andersherum wurden 130 Betroffene (12,3\%) durch die re:set!-Fachstellen in verschiedene andere Versorgungsstrukturen weitervermittelt. Tabelle 2 gibt einen Überblick über die genutzten Beratungsangebote vor der Teilnahme am re:set!-Projekt und die Weitervermittlung durch re:set!-Fachkräfte.

\section{Genutzte Internetanwendungen}

Zu den präferierten Internetanwendungen liegen AICA-S short (Fachverband Medienabhängigkeit e.V., 2010) Daten von 444 Klient_innen vor. Diese Zahl ist geringer als die Gesamtzahl an Klient_innen, um die es in der Beratung ging, da die AICA-S short nur von den Betroffenen selber, und nicht von Angehörigen oder anderen Beratungsaufsuchenden, die ohne die betroffene Person kamen, ausgefüllt wurde. Laut AICA-S short wurden Onlinespiele am häufigsten genutzt $(n=219 ; 49,3 \%)$, gefolgt von Video- oder Streamingportalen $(n=77 ; 17,3 \%)$ und Chatten/dem Austausch in Foren $(n=38 ; 8,6 \%)$. OnlineGlücksspiel $(n=24 ; 5,4 \%)$, Online-Einkaufen $(n=16$; 
$3,6 \%)$ und Online-Erotikangebote $(n=15 ; 3,4 \%)$ sowie die Nutzung von Online-Communities $(n=15 ; 3,4 \%)$ wurden seltener angegeben. Noch seltener wurde die Nutzung anderer Internetspiele $(n=14 ; 3,2 \%)$, Informationsrecherche $(n=10 ; 2,3 \%)$, allgemeines Surfen $(n=6$; $1,4 \%)$ und E-Mail-Nutzung ( $n=1 ; 0,2 \%)$ genannt. Neun Betroffene (2,0\%) gaben andere Anwendungen an, ohne dies weiter zu spezifizieren.
Tabelle 3 zeigt die Ergebnisse des Geschlechtervergleichs bezogen auf die präferierten Internetanwendungen in der AICA-S short (Fachverband Medienabhängigkeit e.V., 2010). Zunächst ist festzustellen, dass der Anteil weiblicher Betroffener sehr gering war. Bei drei Anwendungen wurde auf die Teststatistik verzichtet, da hier keine Angaben von weiblichen Betroffenen vorlagen. Die Ergebnisse deuten auf Geschlechtsunterschiede bezogen auf

Tabelle 2. Beratung vor und Vermittlung nach der Teilnahme am re:set!-Projekt

\begin{tabular}{lcc}
\hline Ort/Person & $\begin{array}{l}\text { Vorherige Beratung } \\
(N=87)\end{array}$ & $\begin{array}{l}\text { Weitervermittlung } \\
(N=130)\end{array}$ \\
\hline Andere Beratungsstelle; $n$ (\%) & $30(34,5)$ & $14(21,5)$ \\
Psychotherapeut_in/Psychiater_in; $n$ (\%) & $25(28,7)$ & $11(8,5)$ \\
Stationäre Rehabilitation; $n$ (\%) & $9(10,3)$ & $16(12,3)$ \\
Ambulante Rehabilitation; $n$ (\%) & - & $2(1,5)$ \\
Jugendhilfe/Jugendamt; $n(\%)$ & $8(9,2)$ & $2(1,5)$ \\
Hausarzt/Hausärztin; $n$ (\%) & $3(3,4)$ & $37(28,5)$ \\
Fachklinik; $n$ (\%) & - & $6(4,6)$ \\
Selbsthilfegruppe; $n$ (\%) & - & $14(10,8)^{\mathrm{b}}$ \\
Sonstige; $n$ (\%) & $12(13,8)^{\mathrm{a}}$ & \\
\hline
\end{tabular}

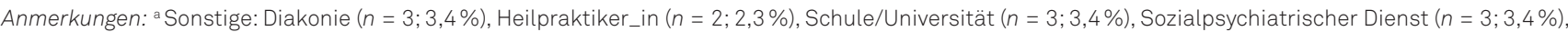

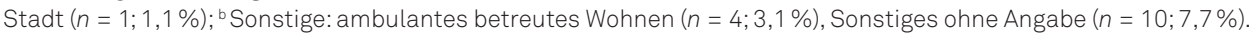

Tabelle 3. Geschlechtsunterschiede bei präferierten Internetanwendungen nach AICA-S short (Fachverband Medienabhängigkeit e.V., 2010)

\begin{tabular}{|c|c|c|c|}
\hline \multirow{2}{*}{$\begin{array}{l}\text { Kategorie der AICA-S short } \\
\text { (Fachverband Medienabhängigkeit e.V., 2010) }\end{array}$} & \multicolumn{2}{|c|}{ Geschlecht der Betroffenen } & \multirow[t]{2}{*}{ Teststatistik } \\
\hline & $\begin{array}{l}\text { Männlich } \\
N=405\end{array}$ & $\begin{array}{l}\text { Weiblich } \\
N=39\end{array}$ & \\
\hline Onlinespiele; $n(\%)$ & $212(52,3)$ & $7(17,9)$ & $X^{2}(1)=13.68 ; p<0.0001 ; \phi=-0.11$ \\
\hline Einkaufen; $n(\%)$ & $10(2,5)$ & $6(15,4)$ & $X^{2}(1)=14.08 ; p=0.003 ; \phi=0.12^{a}$ \\
\hline Chatten/Austausch in Foren; $n$ (\%) & $31(7,7)$ & $7(17,9)$ & $X^{2}(1)=3.31 ; p=0.088 ; \phi=0.06^{a}$ \\
\hline E-Mails schreiben; n (\%) & $1(0,2)$ & - & - \\
\hline Online-Erotikangebote; $n$ (\%) & $15(3,7)$ & - & - \\
\hline Online-Glücksspiel; n (\%) & $20(4,9)$ & $4(10,3)$ & $X^{2}(1)=1.31 ; p=0.284 ; \phi=0.04^{a}$ \\
\hline Online-Communities; $n$ (\%) & $9(2,2)$ & $6(15,4)$ & $X^{2}(1)=15.68 ; p<0.002 ; \phi=0.12^{a}$ \\
\hline Informationsrecherche; n (\%) & $9(2,2)$ & $1(2,6)$ & $x^{2}(1)=0 ; p=1 ; \phi<0.01^{a}$ \\
\hline Video- oder Streamingportale; $n$ (\%) & $71(17,5)$ & $6(15,4)$ & $X^{2}(1)=0.38 ; p=0.536 ; \phi=-0.02$ \\
\hline Andere Internetspiele; $n(\%)$ & $14(3,5)$ & - & - \\
\hline Allgemeines Surfen; $n$ (\%) & $5(1,2)$ & $1(2,6)$ & $X^{2}(1)=0.32 ; p=0.463 ; \phi=0.02^{a}$ \\
\hline Andere; $n(\%)$ & $8(2,0)$ & $1(2,6)$ & $X^{2}(1)=0.02 ; p=0.607 ; \phi<0.01^{a}$ \\
\hline
\end{tabular}

Anmerkungen: a Fisher-Exakt-Test aufgrund erwarteten Zellhäufigkeiten $<5$ bei über $20 \%$ der Zellen. 
die präferierte Internetanwendung hin, was unserer aus der Literatur hergeleiteten Hypothese entspricht. Obwohl Onlinespiele bei beiden Geschlechtern die am häufigsten genannte Internetanwendung darstellen, ist ein signifikanter Unterschied entsprechend der Hypothese, dass mehr männliche Betroffene diese Anwendung nutzen, erkennbar. Auch Online-Erotikangebote wurden eher von männlichen Betroffenen genutzt, während weibliche Betroffene eher Online-Kaufportale und Online-Communities bevorzugten.

\section{Ergebnisse der Screening-Instrumente}

Die restlichen AICA-S short (Fachverband Medienabhängigkeit e.V., 2010) Items wurden von 460 Klient_innen (43,7\%) beantwortet. Die kategoriale Auswertung der AICA-S short erlaubt eine Einteilung in drei Gruppen: kein Risiko einer INS, moderates Risiko einer INS und hohes Risiko einer INS. In der vorliegenden Stichprobe zeigten $35,4 \%(n=163)$ der Betroffenen kein Risiko, 20,4\% ein moderates $(n=94)$ und $44,1 \%$ ein hohes Risiko einer INS $(n=203)$. Wenn die Befunde zu moderatem und hohem Risiko einer INS gemäß AICA-S short zusammengefasst werden, ergibt sich eine geschätzte Prävalenz für das Risiko einer INS von $64,6 \%$.

Des Weiteren wurde getestet, ob zwischen den Gruppen „Risiko einer Internetnutzungsstörung“ und „kein Risiko einer Internetnutzungsstörung" Unterschiede in der Soziodemographie bestanden. Tabelle 4 zeigt die Ergebnisse der Gruppenvergleiche. Gruppenunterschiede ergaben sich lediglich beim Alter und der Ausbildungssituation, wobei die Effektstärken gering ausfielen.

\section{Diskussion}

Die Ergebnisse verdeutlichen, dass durch das neue, medienspezifische re:set!-Beratungsangebot viele Betroffene erreicht werden konnten. Die Fachstellen der Suchtberatung und -prävention können nicht nur erste Anlaufstellen bei exzessivem Medienkonsum sein, sondern sie stellen auch eine bedeutsame Schnittstelle bei der Nachsorge von Personen mit einer INS dar. Zudem bieten Beratungsangebote eine hilfreiche Unterstützung bei und bis zur Weitervermittlung in andere Behandlungsangebote (Petersen \& Thomasius, 2010). Wie aus der Literatur bekannt (Petersen \& Thomasius, 2010), kommt Angehörigen und anderen Bezugspersonen eine wichtige Rolle zu. Etwa ein Drittel der Beratungsaufsuchenden waren besorgte Angehörige/andere Personen meistens die Mütter. Dies könnte mit der jungen Alters- struktur der Betroffenen zusammenhängen und entspricht den Beratungs- und klinischen Erfahrungen mit dieser Klientel (Beutel, Hoch, Wölfling \& Müller, 2011; Bottel, Dieris-Hirche \& te Wildt, 2019; Petersen et al., 2017; Petersen \& Thomasius, 2010).

Personen mit Migrationshintergrund scheinen in dem Projekt unterrepräsentiert zu sein. Dies passt zu Befunden, die darauf hindeuten, dass Menschen mit Migrationshintergrund und einer Abhängigkeitsproblematik seltener Hilfen aufsuchen als Personen ohne Migrationshintergrund (Haasen, Toprak, Yagdiran \& Kleinemeier, 2001; Hayer, Girndt \& Brosowski, 2020). Die Hintergründe dafür gilt es näher zu untersuchen. In jedem Fall unterstreichen die vorliegenden Resultate noch einmal den Bedarf an weiterer Forschung und geeigneten Initiativen, um den Anteil von Menschen mit Migrationshintergrund in der Beratung zu erhöhen.

Eine große deutsche repräsentative Untersuchung zeigte, dass es keinen Geschlechtseffekt im Auftreten einer INS zu geben scheint (Bischof, Bischof, Meyer, John \& Rumpf, 2013; Rumpf et al., 2014). Dazu passen unsere Resultate, die gleichfalls keine Geschlechtsunterschiede in der Prävalenz vermuten lassen. Hier muss allerdings kritisch angemerkt werden, dass der Frauenanteil in unserer Stichprobe sehr gering war, weswegen die Resultate keinesfalls generalisiert werden können. Der geringe Mädchen-/Frauenanteil deckt sich mit anderen Untersuchungen im Bereich INS (Beutel et al., 2011; Bottel et al., 2019). Es stellt sich Frage, warum weibliche Betroffene so selten im re:set!-Projekt auftauchten und wo sie im Hilfesystem zu finden sind. Eine mögliche Erklärung ist, dass INS bei Frauen häufiger unentdeckt bleiben. So zeigte eine Studie, dass INS bei 94,64\% der untersuchten Frauen $(N=327)$ durch die Behandler_innen übersehen wurden (Scherer et al., 2021). Zudem kann vermutet werden, dass Frauen sich eher aufgrund anderer psychischer Belastungen, die bei weiblichen Betroffenen häufiger zu sein scheinen, in Behandlung begeben und die INS somit in den Hintergrund treten könnte (Scherer et al., 2021; Scherer, Mader, Beutel, Wölfling \& Müller, 2019). Zudem sollte in zukünftigen Projekten untersucht werden, ob andere Variablen, wie z.B. die Art der verwendeten Internetanwendung, Geschlechtsunterschiede in den Prävalenzen beeinflussen könnten (Kuss, Griffiths, Karila \& Billieux, 2014). Hinsichtlich der bevorzugten Nutzung der verschiedenen Internetanwendungen ist aus anderen Untersuchungen ein Geschlechterunterschied bekannt (Kuss et al., 2014; Rumpf et al., 2014, Dufour et al., 2016, Petersen et al., 2017). Die am häufigsten genutzte Internetanwendung in der vorliegenden Stichprobe sind Onlinespiele, was sich mit anderen Daten deckt (Bottel et al., 2019; Petersen \& Thomasius, 2010), gefolgt von Videooder Streamingportalen. Die hohe Nutzungsprävalenz 
Tabelle 4. Gruppenvergleiche mit/ohne Risiko einer Internetnutzungsstörung

\begin{tabular}{|c|c|c|c|}
\hline \multirow[t]{2}{*}{ Variable } & \multicolumn{2}{|c|}{ Internetnutzungsstörung } & \multirow[t]{2}{*}{ Teststatistik } \\
\hline & Ja $n(\%)$ & Nein $n(\%)$ & \\
\hline \multicolumn{4}{|l|}{ Geschlecht } \\
\hline Männlich & $270(90,9)$ & $148(90,8)$ & \multirow[t]{2}{*}{$X^{2}(1)<0.01 ; p=0.968 ; \phi<0.01$} \\
\hline Weiblich & $27(9,1)$ & $15(79,2)$ & \\
\hline \multicolumn{4}{|l|}{ Alter [Jahre] } \\
\hline MW & 24.90 & 20.82 & \multirow{3}{*}{$U=16872.00 ; p<0.001 ; \eta_{p}^{2}=0.06$} \\
\hline SD & 10.60 & 9.73 & \\
\hline Range & $10-69$ & $11-64$ & \\
\hline \multicolumn{4}{|l|}{ Muttersprache } \\
\hline Deutsch & $282(95,9)$ & $154(94,5)$ & \multirow[t]{2}{*}{$X^{2}(1)=0.50 ; p=0.481 ; \phi=-0.03$} \\
\hline Andere & $12(4,1)$ & $9(5,5)$ & \\
\hline \multicolumn{4}{|l|}{ Partnerschaft } \\
\hline Mit Partner_in & $66(22,3)$ & $31(19,4)$ & \multirow[t]{2}{*}{$X^{2}(1)=0.53 ; p=0.467 ; \phi=0.03$} \\
\hline Ohne Partner_in & $230(77,7)$ & $129(80,6)$ & \\
\hline \multicolumn{4}{|l|}{ Wohnsituation } \\
\hline Allein lebend & $60(21,3)$ & $18(11,5)$ & \multirow{6}{*}{$X^{2}(1)=15.48 ; p=0.008 ; \phi=0.19$} \\
\hline Mit beiden Eltern lebend & $43(15,2)$ & $31(19,7)$ & \\
\hline Mit einem Elternteil lebend & $28(9,9)$ & $17(10,8)$ & \\
\hline Mit Geschwistern lebend & $66(23,4)$ & $47(29,9)$ & \\
\hline Mit Partner_in lebend & $46(16,3)$ & $13(8,3)$ & \\
\hline Andere & $39(13,8)$ & $31(19,7)$ & \\
\hline \multicolumn{4}{|l|}{ Ausbildungssituation } \\
\hline Aktuell in Ausbildung/Schule/Universität & $146(49,2)$ & $111(68,1)$ & \multirow{2}{*}{$X^{2}(1)=15.31 ; p<0.001 ; \phi=-0.18$} \\
\hline Nicht in Ausbildung/Schule/Universität & $151(50,8)$ & $52(31,9)$ & \\
\hline \multicolumn{4}{|l|}{ Besuch der Ausbildungsstätte } \\
\hline Regelmäßig & $104(74,8)$ & $92(86,0)$ & \multirow[t]{2}{*}{$X^{2}(1)=4.65 ; p=0.031 ; \phi=-0.14$} \\
\hline Unregelmäßig & $35(25,2)$ & $15(14,0)$ & \\
\hline \multicolumn{4}{|l|}{ Schulabschluss } \\
\hline Abitur & $79(36,4)$ & $27(32,1)$ & \multirow{5}{*}{$X^{2}(1)=3.15 ; p=0.533 ; \phi=0.10$} \\
\hline Realschulabschluss & $63(29,0)$ & $28(33,3)$ & \\
\hline Hauptschulabschluss & $59(27,2)$ & $25(29,8)$ & \\
\hline Sonderschulabschluss & $6(2,8)$ & - & \\
\hline Ohne & $10(4,6)$ & $4(4,8)$ & \\
\hline \multicolumn{4}{|l|}{ Erwerbstätigkeit } \\
\hline Arbeitssuchend & $60(32,8)$ & $23(32,4)$ & \multirow{4}{*}{$X^{2}(1)=1.50 ; p=0.682 ; \phi<0.08$} \\
\hline Teilzeit & $18(9,8)$ & $4(5,6)$ & \\
\hline Vollzeit & $67(36,6)$ & $30(42,3)$ & \\
\hline Andere & $38(20,8)$ & $14(19,7)$ & \\
\hline
\end{tabular}

von dem bislang noch wenig erforschten Gebiet Streamingangebote war zunächst etwas überraschend. Diese Anwendung sollte in zukünftigen Forschungsprojekten explizit erfragt werden, da bisher kaum empirische Daten vorliegen. Andere Applikationen, wie z.B. OnlineGlücksspiel oder Online-Einkaufsportale wurden relativ selten genutzt. Hier ist jedoch zu beachten, dass die Beratung bei exzessivem Online-Glücksspiel in den meisten Fachstellen in den Zuständigkeitsbereich der Kolleg_innen fiel, die sich mit Glücksspielsucht generell beschäftigten. Daher kamen die Betroffenen nur ausnahmsweise ins re:set!-Projekt. Bei der Nutzung von Online-Einkaufsportalen ist zu bedenken, dass die recht niedrige Prävalenz auf die geringe weibliche Fallzahl zurückzuführen sein könnte.

Die Interpretation der vorliegenden Ergebnisse unterliegt einigen Limitationen. Die AICA-S short (Fachverband Medienabhängigkeit e.V., 2010) wurde nur einmalig zu Beginn der Beratung ausgefüllt, sodass keine Aussagen zum Symptomverlauf oder zur Effektivität der Beratung getroffen werden können. Eine weitere Limitation ist, dass die Anzahl der ausgefüllten AICA-S short 
Fragebögen stark von der deutlich größeren Gesamtzahl der Beratungsaufsuchenden variiert. Dies hängt damit zusammen, dass die Screening-Bögen ausschließlich von den Betroffenen selber ausgefüllt wurden, die allerdings nur einen Teil der Beratungsaufsuchenden ausmachten. Basierend auf der kategorialen Auswertung der AICA-S short (Fachverband Medienabhängigkeit e.V., 2010) ergaben sich bei mehr als der Hälfte dieser Teilstichprobe Hinweise für eine INS, was mit anderen Studien vergleichbar ist (Beutel et al., 2011). In der deutschen Suchthilfestatistik lag der Anteil der Personen mit der Hauptdiagnose exzessive Mediennutzung zwar bei nur 1\% (Dauber et al., 2019), wobei hier die Suchthilfe im Allgemeinen erfasst wurde, wohingegen das re:set!-Projekt ein spezifisches Beratungsangebot für exzessive Mediennutzung war.

Der Altersunterschied zwischen den Beratungsaufsuchenden mit Risiko und ohne Risiko für eine INS sollte aufgrund der sehr jungen, selektiven Stichprobe und der geringen Effektstärke dieses Gruppenunterschieds nicht überbewertet werden. Obwohl hier die Betroffenen mit Risiko für eine INS älter waren, zeigen repräsentative Bevölkerungsstudien eine erhöhte Prävalenz von INS in den jüngeren Altersgruppen (Bischof et al., 2013; Rumpf, et al., 2014, 2016).

\section{Schlussfolgerungen für die Praxis}

- Fachstellen für Suchtberatung und -prävention sind eine wichtige Anlaufstelle für Betroffene mit exzessivem Medienkonsum und deren Angehörige. Sie können sowohl als erste Anlaufstelle fungieren, als auch Betroffene mit einer Internetnutzungsstörung weitervermitteln.

- Hinsichtlich der präferierten Internetanwendung der Beratungsaufsuchenden zeigten sich die bereits aus der Literatur bekannten Geschlechtseffekte.

- Bei mehr als der Hälfte der Beratungsaufsuchenden ergaben sich Hinweise auf das Vorliegen einer Internetnutzungsstörung.

- Bezogen auf sozioökonomischen Status, Bildung, Herkunft und Geschlecht konnten keine relevanten Unterschiede zwischen Betroffenen mit und ohne Internetnutzungsstörung gefunden werden.

- Es bedarf geeigneter Initiativen, um sowohl den Mädchen-/Frauenanteil als auch den von Personen mit Migrationshintergrund in der Beratung zu erhöhen.

- Projekte wie re:set! sollten verstetigt werden, um die Versorgung für Menschen mit Internetnutzungsstörungen zu verbessern.

\section{Literatur}

Ballester-Arnal, R., Castro Calvo, J., Gil-Llario, M.D. \& Gil-Julia, B. (2017). Cybersex Addiction: A Study on Spanish College Students. Journal of Sex and Marital Therapy, 43 (6), 567-585. https://doi.org/10.1080/0092623X.2016.1208700

Beutel, M.E., Hoch, C., Wölfling, K. \& Müller, K.W. (2011). Klinische Merkmale der Computerspiel- und Internetsucht am Beispiel der Inanspruchnehmer einer Spielsuchtambulanz. Zeitschrift für Psychosomatische Medizin und Psychotherapie, 57 (1), $77-$ 90. https://doi.org/10.13109/zptm.2011.57.1.77

Bischof, G., Bischof, A., Meyer, C., John, U. \& Rumpf, H.-J. (2013). Prävalenz der Internetabhängigkeit - Diagnostik und Risikoprofile (PINTA-DIARI). Kompaktbericht an das Bundesministerium für Gesundheit, Universität zu Lübeck.

Bottel, L., Dieris-Hirche, J. \& te Wildt, B. (2019). Personenbezogene Merkmale von Teilnehmenden des Online-Ambulanz-Service für Internetsüchtige (OASIS). Suchttherapie, 20 (S 01). https:// doi.org/10.1055/s-0039-1696259

Brand, M., Rumpf, H., Demetrovics, Z., Müller, A., Stark, R., King, D.L. et al. (2020). Which conditions should be considered as disorders in the International Classification of Diseases (ICD11) designation of "other specified disorders due to addictive behaviors"? Journal of Behavioral Addictions. Advance online publication. https://doi.org/10.1556/2006.2020.00035

Cohen, J. (1988). Statistical power analysis fot the behavioral sciences (2nd ed.). Hillsdale, NJ: Lawrence Erlbaum Associates, Publishers.

Dauber, H., Specht, S., Künzel, J., Pfeiffer-Gerschel, T. \& Braun, B. (2019). Suchthilfe in Deutschland 2018: Jahresbericht der Deutschen Suchthilfestatistik (DSHD). München: IFT Institut für Therapieforschung.

Dufour, M., Brunelle, N., Tremblay, J., Leclerc, D., Cousineau, M.-M., Khazaal, Y. et al. (2016). Gender difference in Internet use and Internet problems among Quebec high school students. Canadian Journal of Psychiatry, 61 (10), 663-668. https://doi.org./10. 1177/0706743716640755

Fachverband Medienabhängigkeit e.V. (Hrsg.). (2010). Assessment for Internet and Computer Game Addiction Scale (AICA-S short). Verfügbar unter https://www.fv-medienabhaengigkeit.de/file admin/images/Dateien/AICA-Sshort_2017_FVMA.pdf

Haasen, C., Toprak, M.A., Yagdiran, O. \& Kleinemeier, E. (2001). Psychosoziale Aspekte der Sucht bei Migraten. Suchttherapie, 2 (3), 161-166. https://doi.org/10.1055/s-2001-16409

Hayer, T., Girndt, L. \& Brosowski, T. (2020). Die Bedeutung der Selbsthilfe in der Versorgung pathologischer Glücksspieler*innen: Nutzen, Grenzen und Optimierungspotenziale. Bremen: Universität Bremen.

Kuss, D.J., Griffiths, M.D., Karila, J. \& Billieux, J. (2014). Internet Addiction: A systematic review of epidemiological research for the last decade. Current Pharmaceutical Design, 20 (25), 4026 4052. https://doi.org/10.2174/13816128113199990617

Laskowski, N.M., Meyer, V., Trillmich, T., Ernst, A., \& Müller, A. (2020). Empfehlungen zur Implementierung eines Beratungsangebots bei exzessivem Medienkonsum und internetbezogenen Störungen. Suchttherapie, 22, 44-51. https://doi.org/10. 1055/a-1234-1644

Maraz, A., Griffiths, M.D., \& Demetrovics, Z. (2016). The prevalence of compulsive buying: a meta-analysis. Addiction, 111 (3), 408419. https://doi.org/10.1111/add.13223

McCormack, A., Shorter, G.W., \& Griffiths, M.D. (2014). An empirical study of gender differences in online gambling. Journal of GamblingStudies 30 (1), 71-88. https://doi.org/10.1007/s10899-0129341-x

Müller, A., Brand, M., Claes, L., Demetrovics, Z., de Zwaan, M., Fernández-Aranda, F. et al. (2019). Buying-shopping disorder - 
is there enough evidence to support its inclusion in ICD-11? CNS Spectrums, 24 (4), 374-379. https://doi.org/10.1017/ S1092852918001323

Müller, A., Ernst, A., Meyer, V., Jones, E. \& Laskowski, N. M. (2020). Dokumentation und Inanspruchnahme der medienspezifischen Beratung in den re:set!-Fachstellen für Sucht und Suchtprävention. Hannover: Niedersächsische Landesstelle für Suchtfragen.

Müller, A., Meyer, V., Ernst, A. \& Laskowski, N.M. (2020). Exzessiver Medienkonsum: Beratungsleitfaden mit Materialsammlung. Hannover: Niedersächsische Landesstelle für Suchtfragen.

Müller, A., Meyer, V., Schwenk, V. \& Laskowski, N. M. (2019). Empfehlungen zur Implementierung eines Beratungsangebots bei exzessivem Medienkonsums und internetbezogenen Störungen. Hannover: Niedersächsische Landesstelle für Suchtfragen.

Ostendorf, S., Wegmann, E. \& Brand, M. (2020). Problematic socialnetworks-use in German children and adolescents - the interaction of need to belong, online self-regulative competences, and age. International Journal of Environmental Research and Public Health 17 (7), 2518. https://doi.org/10.3390/ijerph 17072518

Petersen, K.U. \& Thomasius, R. (2010). Beratungs- und Behandlungsangebote zum pathologischen Internetgebrauch in Deutschland. Lengerich: Pabst Science Publisher.

Petersen, K.U., Hanke, S., Bieber, L., Mühleck, A. \& Batra, A. (2017). Angebote bei internetbasiertem Suchtverhalten. Lengerich: Pabst Science Publishers.

Petry, N., Stinson, F.S. \& Grant, B.F. (2005). Comorbidity of DSM-IV pathological gambling and other psychiatric disorders: results from the National Epidemiologic Survey on Alcohol and Related Conditions. Journal of Clinical Psychiatry, 66 (5), 564-574. https://doi.org/10.4088/jcp.v66n0504

Rehbein, F., Kliem, S., Baier, D., Mößle, T. \& Petry, N. M. (2015). Prevalence of Internet gaming disorder in German adolescents: diagnostic contribution of the nine DSM- 5 criteria in a state-wide representative sample. Addiction, 110 (5), 842-851. https://doi. org/10.1111/add.12849

Rumpf, H.-J., Arnaud, N., Batra, A., Bischof, A., Bischof, G., Brand, M. et al. (2016). Memorandum Internetbezogene Störungen der Deutschen Gesellschaft für Suchtforschung und Suchttherapie (DG-Sucht). SUCHT, 62 (3), 167-172. https://doi.org/10.1024/ 0939-5911/a000425

Rumpf, H.-J., Vermulst, A.A., Bischof, A., Kastirke, N., Gürtler, D., Bischof, G. et al. (2014). Occurence of internet addiction in a general population sample: a latent class analysis. European Addiction Research, 20 (4), 159-166. https://doi.org/10.1159/ 000354321
Scherer, L., Mader, L., Beutel, M. E., Wölfling, K. \& Müller, K.W. (2019). Geschlechtsspezifische Aspekte der Internetsucht. Suchttherapie, 20 (S01). https://doi.org/10.1055/s-0039-1696222

Scherer, L., Mader, L., Wölfling, K., Beutel, M., Dieris-Hirche, J. \& Müller, K.W. (2021). Nicht diagnostizierte internetbezogene Störungen im psychotherapeutischen Versorgugssystem: Prävalenz und geschlechtsspezifische Besonderheiten. Psychiatrische Praxis. Advance online publication. https://doi.org/10. 1055/a-1364-8396

Wedegärtner, F. \& Wedegärtner, C. (2010). Behandlungssituation und Behandlungskosten von Medienabhängigkeit und pathologischem Glücksspiel in Deutschland. In D. Mücken, A. Teske, F. Rehbein \& B. te Wildt (Hrsg.), Prävention, Diagnostik und Therapie von Computerspielabhängigkeit (S. 101-113). Lengerich: Pabst Science Publishers.

Wölfling, K., Beutel, M.E. \& Müller, K.W. (2016). OSV-S - Skala zum Onlinesuchtverhalten. In K. Geue, B. Strauß \& E. Brähler (Hrsg.), Diagnostische Verfahren in der Psychotherapie (Diagnostik für Klinik und Praxis) (S. 362-366). Göttingen: Hogrefe.

World Health Organisation (WHO). (2019). International Classification of Diseases, updated 09/2019 (11th revision). Verfügbar unter https://icd.who.int/en/

\section{Historie}

Manuskript eingereicht: 03.03.2021

Manuskript angenommen: 13.08.2021

\section{Danksagung}

Unser Dank gilt den am Projekt beteiligten Fachkräften sowie dem Niedersächsischen Ministerium für Soziales, Gesundheit und Gleichstellung, welches dieses Projekt gefördert hat.

\section{Deklaration konkurrierender Interessen}

Keine

\section{Förderung}

Open-Access-Veröffentlichung ermöglicht durch Medizinische Hochschule Hannover (MHH).

\section{Dr. Nora M. Laskowski}

Klinik für Psychosomatik und Psychotherapie Medizinische Hochschule Hannover

Carl-Neuberg-Straße 1

30625 Hannover

Deutschland

laskowski.nora@mh-hannover.de 